\title{
In vivo MRI visualization of mesh shrinkage using surgical implants loaded with superparamagnetic iron oxides
}

\author{
Nicolas Kuehnert • Nils A. Kraemer • Jens Otto • \\ Hank C. W. Donker • Ioana Slabu • Martin Baumann • \\ Christiane K. Kuhl • Uwe Klinge
}

Received: 29 May 2011/Accepted: 8 November 2011/Published online: 17 December 2011

(C) The Author(s) 2011. This article is published with open access at Springerlink.com

\begin{abstract}
Background Prosthetic mesh implants are widely used in hernia surgery. To show long-term mesh-related complications such as shrinkage or adhesions, a precise visualization of meshes and their vicinity in vivo is important. By supplementing mesh fibers with ferro particles, magnetic resonance imaging (MRI) can help to delineate the mesh itself. This study aimed to demonstrate and quantify timedependent mesh shrinkage in vivo by MRI.

Methods Polyvinylidenfluoride (PVDF) meshes with incorporated superparamagnetic iron oxides (SPIOs) were implanted as an abdominal wall replacement in 30 rats. On days $1,7,14$, or 21 , MRI was performed using a gradient echo sequence with repetition time (TR)/echo time (TE) of $50 / 4.6$ and a flip angle of $20^{\circ}$. The length, width, and area of the device were measured on axial, coronal, and sagittal images, and geometric deformations were assessed by surgical explantation.

Results In all cases, the meshes were visualized and their area estimated by measuring the length and width of the mesh. The MRI presented a mean area shrinkage in vivo of
\end{abstract}

Nicolas Kuehnert and Nils A. Kraemer contributed equally to this study.

N. Kuehnert $(\bowtie) \cdot$ J. Otto $\cdot$ U. Klinge

Department of Surgery, University Hospital, RWTH Aachen

University, 52074 Aachen, Germany

e-mail: nkuehnert@ukaachen.de

N. A. Kraemer · H. C. W. Donker - C. K. Kuhl

Department of Diagnostic Radiology, University Hospital,

RWTH Aachen University, 52074 Aachen, Germany

I. Slabu · M. Baumann · U. Klinge

Helmholtz Institute for Applied Medical Engineering, RWTH

Aachen University, 52074 Aachen, Germany
$13 \%$ on day $7,23 \%$ on day 14 , and $23 \%$ on day 21 . Postmortem measurements differed statistically from MRI, with a mean area shrinkage of $23 \%$ on day $7,28 \%$ on day 14 , and $30 \%$ on day 21 . Ex vivo measurements of shrinkage showed in vivo measurements to be overestimated approximately $8 \%$. Delineation of the mesh helped to show folding or adhesions close to the intestine.

Conclusion Loading of surgical meshes with SPIOs allows their precise visualization during MRI and guarantees an accurate in vivo assessment of their shrinkage. The authors' observation clearly indicates that shrinkage in vivo is remarkably less than that shown by illustrated explantation measurements. The use of MRI with such meshes could be a reliable technique for checking on proper operation of implanted meshes and showing related complications, obviating the need for exploratory open surgical revision.

Keywords Magnetic resonance imaging - SPIO · Mesh implant · Shrinkage

Treatment of abdominal hernias is one of the most frequently performed operations annually worldwide, either by open surgery or by an increasing percentage of laparoscopy procedures [1]. Reinforcement of tissues with mesh implants is definitely the treatment of choice due to reports of low recurrence rates, ease of use, and low morbidity rates [2-4].

Despite the increasing popularity of prosthetic mesh for hernia repair, the long-term complications after intraperitoneal implantation remain a concern [5]. A substantial number of studies report mesh-associated complications such as seromas, bacterial infections, chronic pain, adhesions, and mesh shrinkage. These complications may 
demand surgical revision, with the removal or correction of the implanted device [5-9]. Furthermore, for up to $70 \%$ of patients with recurrent hernia, the surgeon must take into account that the previous treatment was performed with a mesh, which currently is somewhere in the abdominal wall $[10,11]$ and could complicate further treatment or lead to elaborate explantation.

As all conventional radiological methods fail in precise visualization of meshes, almost any reliable clinical information can be drawn without precise mesh delineation $[6,12-16]$. Therefore, surgical exploration is the inevitable consequence. To overcome this problem, the integration of superparamagnetic iron oxides (SPIOs) into polymer-based implants combined with magnetic resonance imaging (MRI) has been proposed for delineation of these otherwise invisible implants [17]. Using this new approach, the mesh implant can be delineated as hypointense (dark) susceptibility artifacts against the hyperintense (bright) surrounding tissue, particularly the intraabdominal fat.

In this study, the new visible mesh was evaluated using MRI in an animal model. This evaluation aimed to assess mesh shrinkage in vivo and to compare the MRI measurements with explanted meshes as the gold standard.

\section{Methods}

Place of study

The study was performed at the Institute for Laboratory Animal Science (ILAS) of the University Hospital in Aachen (UK-Aachen, Germany).

\section{Sampling}

For the study, 30 male Sprague-Dawley rats weighing approximately 300 to $450 \mathrm{~g}$ at surgery were housed under conditions of constant light, temperature, and humidity. The animals received a complete diet of rat chow and water ad libitum throughout the entire study, which was performed according to the rules of the German Tierschutzgesetz, Sec. 8 Abs. 1, and in accordance with the Guide for the Care and Use of Laboratory Animals published by the National Institutes of Health (NIH). The animals were divided into three groups according to three different observation periods. After 2 weeks of acclimatization time, the laboratory animals had gained an optimal weight.

Prosthetic material

Polyvinylidenfluoride (PVDF) was used as the base material of the prosthetic mesh. To provide MR contrast by induction

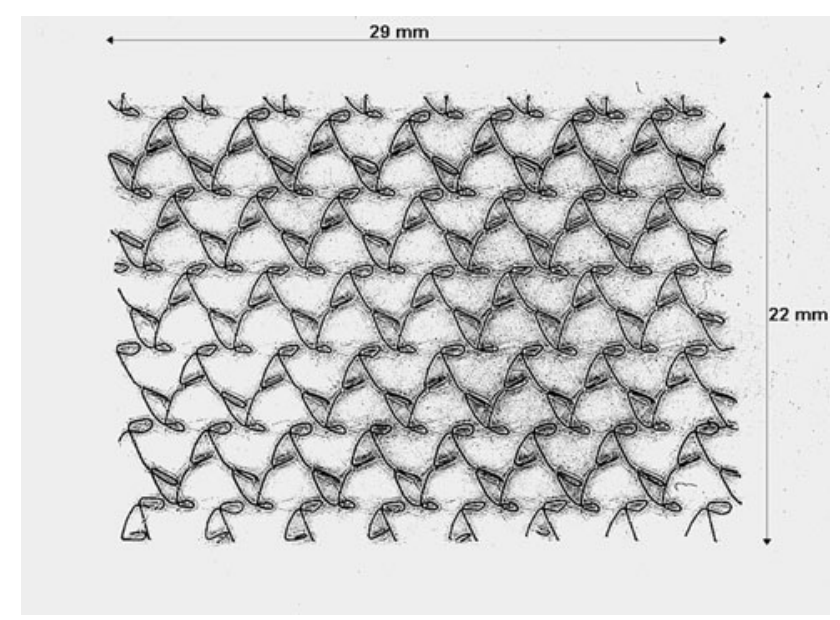

Fig. 1 Polyvinylidenfluoride (PVDF) meshes $(22 \times 29 \mathrm{~mm})$ loaded with superparamagnetic iron oxides (SPIOs)

of susceptible artifacts, this base material was dotted with SPIOs in a concentration of $10 \mathrm{mg} / \mathrm{g}$, as previously described [17]. After extrusion of filaments with a diameter of $85 \mu \mathrm{m}$, visible meshes size $22 \times 29 \mathrm{~mm}$ were assembled by FEG Textiltechnik (Aachen, Germany) (Fig. 1).

Surgical procedure

After balanced anesthesia with inhalation gas isoflurane and subcutaneous injection of Ketamin/Domitor suspension, the desired anesthetic depth was achieved. The anterior abdominal wall was shaved, disinfected with a polividione-iodine solution, and covered with sterile perforated sheets. After a midline incision $2 \mathrm{~cm}$ distal to the xiphoid, which served as the orientation point, a fullthickness defect about $2 \times 3 \mathrm{~cm}$ in size was resected en bloc including the rectus muscle and the peritoneum.

The SPIO-loaded mesh was fixed as an abdominal wall replacement in a cranial-caudal direction. Continuous suture using prolene $5 / 0$ facilitated mesh fixation without overlap between muscle and biomaterial, as shown in Fig. 2. The meshes were fixed without wrinkles that could result in a loss of area. Skin closure was finally obtained by silk 3/0 continuous sutures (Multifil/Vicryl). Silver-aluminium aerosol was applied to protect against automutilation and to induce disinfection. No additional antibiotic treatment was given before, during, or after surgery.

\section{Observation period}

The moment of surgery was marked as day 0 . The animals were allowed to recover after surgery in their boxes during the immediate postoperative time, to eat chow, and to drink ad libitum. All the rats underwent MR examinations at two dates. First, MRI examination was performed on day 1 


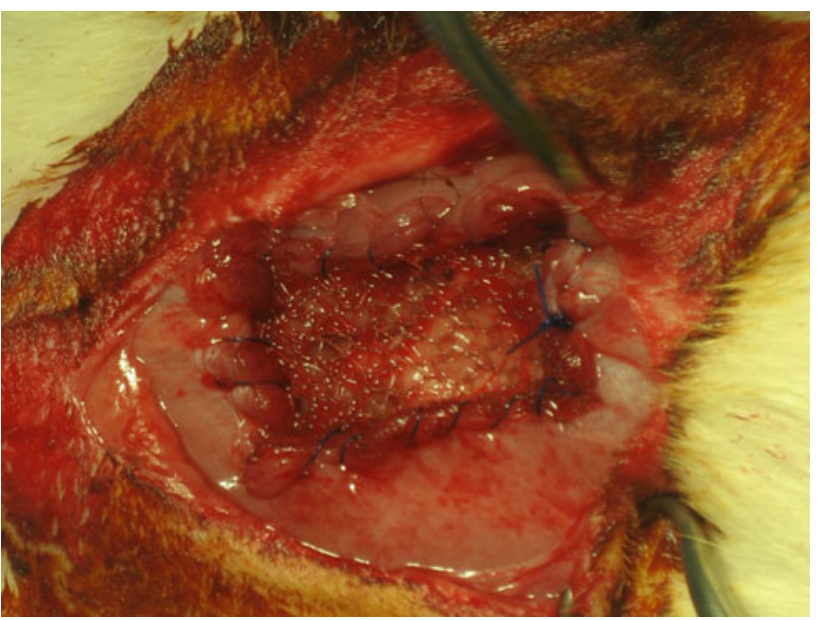

Fig. 2 Fixed mesh as an abdominal wall replacement

postoperatively. Second, MRI was measured after 7, 14, or 21 days depending on the observation period-related group classification (see study group later). Throughout the entire observation period, all the animals were controlled objectively and subjected to daily clinical investigation for assessment of local and systemic complications. Dissection and explantation of implanted mesh took place immediately after the second MRI examination.

\section{Study group}

The 30 animals were divided into three groups (with 10 animals in each), according to the observation period (7, 14, or 21 days). After the second MRI examination, the rats were killed with an overdose of isoflurane gas, and the meshes were explanted immediately.

\section{MRI}

The rats with implanted meshes were examined by MRI with a 1.5-Tesla scanner (Achieve; Philips Healthcare, Best, The Netherlands) using a dedicated small animal micro surface coil (diameter, $47 \mathrm{~mm}$ ). The animals were positioned headfirst, prone, and free-breathing. Conventional gradient echo sequences were acquired in transverse, sagittal, and coronal orientations. The sequence parameters were a repetition time (TR) of $50 \mathrm{~ms}$, an echo time (TE) of $4.6 \mathrm{~ms}$, and a flip angle of $20^{\circ}$. During detection of the abdominal wall, the rats were under anesthesia to prevent moving and resultant artifacts. The average MR scanning time was 45 min.

\section{MRI analysis}

To identify shrinkage of implanted mesh by MRI and to facilitate comparison of these results with the explantation data, we measured in transverse, sagittal, and coronal

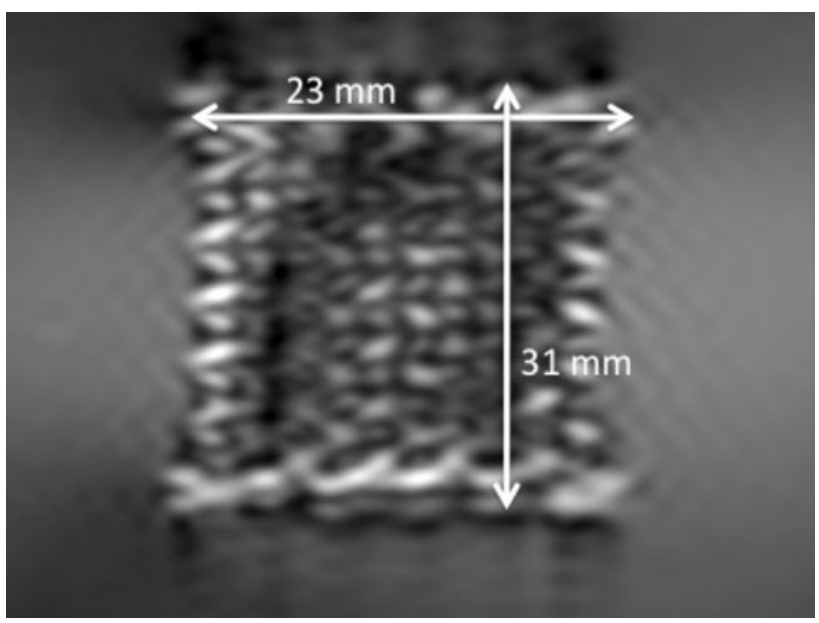

Fig. 3 Phantom measurement of mesh loaded with superparamagnetic iron oxides (SPIOs) using magnetic resonance imaging (MRI)

orientations. The maximal diameters (length and width) of the meshes on the MRI were measured using a DICOMViewer program for MacOS X (Osirix Imaging Software, Osirix v.3.8.1; 32 Bit Medical Systems, Los Angeles, CA, USA). The measurement technique using this software compared with conventional ex vivo measurement procedures, as illustrated in Fig. 3. The DICOM-Viewer program requires a tool for tracing a line precisely over the utmost distension of implanted mesh. To find the maximal distension, it is necessary to measure all slices of MRI, with consideration of precise anterior and posterior views.

To achieve our aim of measuring the largest extent of the implanted mesh, it was necessary to measure several times at different locations to obtain a mean value of the data. The geometric accuracy of all the measurements was important to avoid inaccuracies. The area was estimated by multiplication of length and width. Wrinkles and other geometric deformations were included in the loss of area. Tracing precisely over the contour of mesh wrinkles was not favored, so only the covering area of the mesh was measured.

\section{Explantation technique}

After the animals had been killed with isoflurane in a chamber on the predetermined dates after MRI detection, they were dissected, and wide depiction of the anterior abdominal aponeurosis was used as shown in Fig. 4 to measure the dimensions of the vertical (length) and horizontal (width) edges of the meshes.

Statistical analysis

Data were collected in a database, and statistical analysis was performed using the Statistical Package for Social Science (SPSS Inc., Chicago, IL, USA) for Windows. 


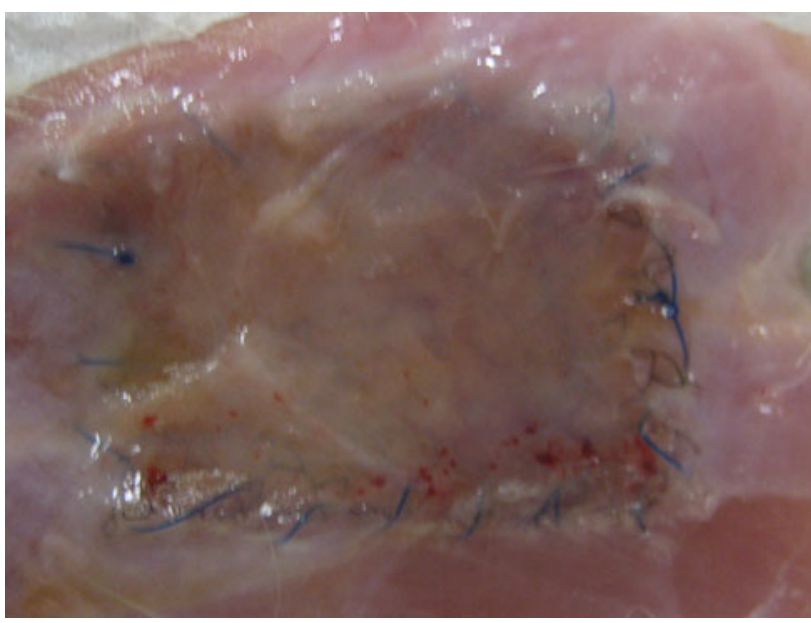

Fig. 4 Explanted mesh with surrounding tissue

All data were presented as mean, minimum, and maximum values. All MRI-measured mesh dimensions were compared with mesh dimensions after explantation using a Pearson correlation coefficient. The statistically significant difference in shrinkage between the two methods was determined using a Wilcoxon matched-pairs, signed-rank test. Statistical significance was considered at $p$ values less than 0.05 .

\section{Results}

After repair of full-thickness wall defect with a mesh, 27 of the 30 rats developed favorably during the postoperative period. Two rats in group 3 died during the first MR detection, likely due to an overdose of anesthesia. One rat in group 1 was terminated because of automutilation after surgery. No mesh-related infections were observed in any case. Bowel obstruction due to intraabdominal adhesions did not manifest.

At the final autopsy, adhesions between the omentum majus and the surgical mesh were found in all the rats. No other mesh-related intestinal complications were found. Microscopic analysis with hematoxylin and eosin (H\&E) staining showed a normal moderate foreign body reaction around the PVDF fibers without any specific differences due to the presence of ferro particles.

MR imaging of mesh in vivo

Via MRI, it was possible to identify the implanted device in all 27 rats in situ. The mesh was delineated as a thin smooth foil abdominal replacement beside the rectus muscle. Details such as the pores of the mesh and the fiber ends sometimes were visible by MR images, but not in all cases. The mesh usually was depicted as a black structure due to its lack of water signals. Correspondingly, the implanted mesh showed a clear contrast to the surrounding muscles (Fig. 5A) and fat tissue (Fig. 5B), whereas it was difficult to separate it from gas of the bowels (Fig. 5C). The mesh position of all the prostheses was meticulously feasible and always found in the place where it had been implanted and where it was found subsequently during excision. Regardless of the alignment in the abdomen, measurements could be performed easily in sagittal, transverse, and coronal image analysis (Fig. 5A-D). No important artifacts presented that impaired the image analysis significantly.

\section{Shrinkage of the mesh}

Over time, the size of the mesh measured ex vivo after dissection of all surrounding tissues decreases compared with the full-scale mesh sizes defined before implantation. The major change in mesh shrinkage occurred already between days 7 and 14, as shown in Tables 1, 2, and 3 . Eventually, after 21 days, the length showed a mean reduction of $22.7 \%$ (range, $13.2-30.6 \% ; p<0.0009$ ) and the width a reduction of 9\% (range, 6.1-10.8\%; $p<0.0009)$. The mean loss in area after 21 days was $29.7 \%$ (range, 18.5-38.0\%; $p<0.0009$ ) (Tables 1, 2, 3), as illustrated in Fig. 5D compared with Fig. 5C, which shows a mesh area shrinkage of $23 \%$.

Similarly, the shrinkage of length and width measured by MRI in vivo occurred between days 7 and 14, as shown in Tables 1, 2, and 3. Compared with full-scale mesh, the length showed a mean shrinkage in vivo of $15.8 \%$ (range, $7.4-32.8 \% ; p<0.003)$ after 21 days, and the width showed a shrinkage of $8.7 \%$ (range, $5.2-14 \%$; $p<0.002$ ). The mean loss of area in vivo after 21 days of observation time was $23 \%$ (range, 12-29\%; $p<0,001$ ).

In vivo and in vitro, the major reduction in mesh area was demonstrated between days 7 and 14, whereas after 21 days, it already seemed to narrow to a plateau.

At all time points, MRI and postmortem measurements differed significantly, as verified with a Wilcoxon matchedpairs, signed-rank test with a $p$ value less than 0.005 . This difference was based on $8 \%$ additional mean shrinkage of tissue postmortem compared with in vivo measurements. This method-specific divergence can be compensated by increasing the mesh area of the explanted meshes by an average of $8 \%$, which leads to a close correlation between MRI and explantation measurements, with a correlation coefficient of $r$ equal to 0.9920 .

\section{Discussion}

Surgical hernia repair is the operation most frequently performed in surgery worldwide [18, 19]. Thereby, 

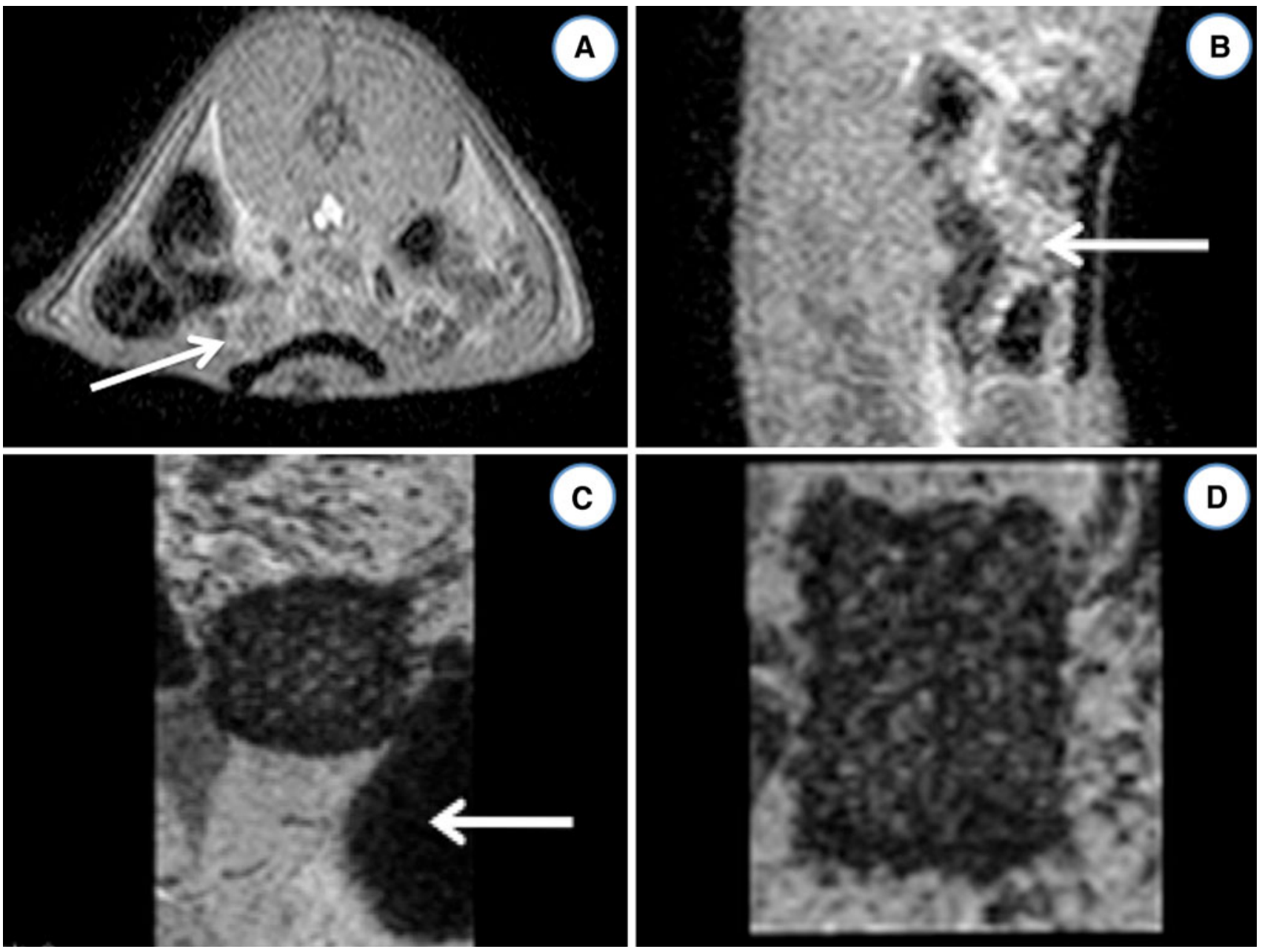

Fig. 5 In vivo measurements of implanted mesh illustrated in three different orientations: A (transversal), B (sagittal), C (coronal), and D (coronal)

Table 1 Mean shrinkage of length in percentage (range)

\begin{tabular}{llll}
\hline & $\begin{array}{l}7 \text { days } \\
(\text { range })\end{array}$ & $\begin{array}{l}14 \text { days } \\
(n=9)\end{array}$ & $\begin{array}{l}21 \text { days } \\
\%=10)\end{array}$ \\
\hline MRI & $11.9(4.9-20.3)$ & $16.5(9.7-26.8)$ & $15.8(7.4-32.8)$ \\
Expl & $15.9(9.7-27.1)$ & $18.8(13.2-23.6)$ & $22.7(13.2-30.6)$ \\
\hline
\end{tabular}

MRI, magnetic resonance imaging; Expl, explantation

Table 2 Mean shrinkage of width in percentage (range)

\begin{tabular}{llll}
\hline & $\begin{array}{l}7 \text { days } \\
(\text { range }) \\
(n=9)\end{array}$ & $\begin{array}{l}14 \text { days } \\
(\text { range }) \\
(n=10)\end{array}$ & $\begin{array}{l}21 \text { days } \\
(\text { range }) \\
(n=8)\end{array}$ \\
\hline MRI & $1.2(0.1-5.4)$ & $8.1(2.3-18.0)$ & $8.7(5.2-14.0)$ \\
Expl & $8.2(6.1-15.5)$ & $11.3(6.1-20.2)$ & $9.0(6.1-10.8)$
\end{tabular}

MRI, magnetic resonance imaging; Expl, explantation
Table 3 Mean shrinkage of area in percentage (range)

\begin{tabular}{|c|c|c|c|}
\hline & $\begin{array}{l}7 \text { days } \\
\% \text { (range) } \\
(n=9)\end{array}$ & $\begin{array}{l}14 \text { days } \\
\% \text { (range) } \\
(n=10)\end{array}$ & $\begin{array}{l}21 \text { days } \\
\% \text { (range) } \\
(n=8)\end{array}$ \\
\hline MRI & $12.9(7.7-18.1)$ & $23.3(14.9-33.3)$ & $23.0(12.9-29.0)$ \\
\hline Expl & $22.7(15.2-35.4)$ & $27.8(18.5-34.3)$ & $29.7(18.5-38.0)$ \\
\hline
\end{tabular}

MRI, magnetic resonance imaging; Expl, explantation

alloplastic materials are implanted routinely [20]. Due to the rising popularity of mesh materials, an increasing number of studies have reported some long-term consequences $[5,21-23]$. One major concern is that the possible shrinkage of these textile structures favors recurrences of hernia and pain [5]. A large number of experimental studies have addressed this important issue [24, 25]. However, the lack of direct mesh surveillance in vivo hinders consistent results for the complex process of shrinkage. Consequently, reliable clinical conclusions are 
almost impossible [7]. A noninvasive diagnostic method for visualizing the mesh size in vivo thus is desirable [16].

Parra et al. [8] already had described ultrasound as an effective, noninvasive, simple diagnostic method for indentifying and mapping abdominal complaints after mesh implantation. However, due to the small size of mesh filaments and their isoecogenity to the surrounding tissue, scanning of a thin prosthetic mesh material becomes very challenging. Thus, in vivo measurements of mesh shrinkage by ultrasound cannot be assessed precisely. Only computed tomography (CT) delineated expanded polytetrafluoroethylene (ePTFE) meshes precisely as a hyperdense line, probably because of their composition and thickness, but it failed to delineate other materials [8]. Because it entails considerable radiation exposure, CT is not appropriate as a routine procedure for every patient or for followup studies. Furthermore, for patients in whom one of the two main axes of implanted mesh was not parallel to the craniocaudal axis of the patient's body, the mesh area could not be defined exactly [5, 6].

The use of MRI was focused on noninvasive identification of incisional hernia repair and implanted synthetic meshes as well as on evaluation of postsurgical complications, particularly adhesions, and also of the mesh itself $[14,16]$. Follow-up studies published initial results, which again showed only the implanted ePTFE mesh visible in MR images, allowing an accurate assessment of the mesh and its fixation [5, 12, 16]. In contrast to the film-like ePTFE, the modern porous meshes did not provide sufficient contrast for either CT or MRI.

To our knowledge, the current study is the first attempt at direct visualization of net-like porous textile structures. It is known that SPIOs enhance contrast in MRI, and thus are added to the spinning of the fibers for the mesh [17, 26, 27]. Polyvinylidenefluoride (PVDF) as a base material of the mesh can be considered as widely inert and largely resistant to bacterial infections, with proven long-term stability [28]. However, it does not show any contrast in its pure form with either CT or MRI.

In this study, PVDF induced a slight inflammatory reaction with only small amounts of fibrotic tissue and was usable even within the abdominal cavity in contact with bowels. Whereas the area of the film-like ePTFE in some studies is reduced to less than to $50 \%$ due to wound contraction of the fibrotic capsule, it is significantly decreased to less than $30 \%$ if large-pore flat meshes are used instead [3, 29, 30].

The mesh constructed for this experiment had a structure with some elasticity mainly in a vertical direction (right angled to the course of the fibers), whereas due to the manufacturing process, the stretchability and shortening in a horizontal direction was limited (FEG Textiltechnik Forschung- und Entwicklungsgesellschaft mbH, Aachen,
Germany). This anisotropic elasticity is reflected by the measurements of shrinkage, which is greater for the length and significantly less for the width. This finding is consistent in MRI and ex situ measurements, which both confirm that the extent of shrinkage is strongly affected by the textile structure of the implant.

Unfortunately, although this anisotropy of the surgical meshes is apparent for most of the devices, it rarely is considered in examination of shrinkage. Further studies must show which mesh properties prevent the best shrinkage and simultaneously provide elasticity.

Embedding SPIOs in PVDF-based mesh allows both optimal localization of implanted surgical mesh and inspection of tissue in the direct vicinity of these implants. The excellent resolution of the MRI allows even depiction of the contrast-enhanced pores between the filaments supplemented with SPIOs [17].

Phantom measurements of PVDF meshes loaded with SPIOs showed a $6 \%$ overestimation by MR imaging in contrast to the original sizes of this mesh, as illustrated by Fig. 1 versus Fig. 3. This indicates a maximum overestimation of $2 \mathrm{~mm}$ by MRI, which remains constant even if much larger meshes are used. This MRI-specific inaccuracy is irrelevant in clinical application because $2 \mathrm{~mm}$ is negligible concerning conventional mesh sizes. In contrast, dissection of the mesh from all surrounding tissue leads to further reduction of the mesh size ex situ, falsely overrating shrinkage by almost $8 \%$. This fact is well known for all distance measurements in excised tissue (e.g., distance of the tumor's bowel resection leads to an overestimation of shrinkage) [31, 32]. It is in line with studies that published shortening of sarcomer length by rigor mortis of about $11 \%$ within $3 \mathrm{~h}$ postmortem [33]. Nevertheless, comparison of ex situ measurements with the MRI measurements showed high correlation at all times of investigation.

A possibility of visualizing the correct placement of a mesh device with sufficient contrast to surrounding tissue such as seroma, hematoma, bowels, or other organs should help to avoid unnecessary revision operations. The current study showed that the use of SPIOs in mesh fibers allows differentiation even between surgical mesh and bowel. Thus, these meshes can be helpful, particularly for areas in which the identification of meshes is not as clear as in the abdominal wall (e.g., in the hiatus or the pelvic floor, both of which have a high risk of mesh migration).

A limitation of our study was the small dimension of the applied mesh in rats with considerable influence of the surgical technique. Furthermore, the MRI sequences were optimized for small laboratory animals and must be adapted to larger animals and humans. The short observation period reaching only 3 weeks may be too short for the long-term extent of shrinkage to be evaluated. 


\section{Conclusion}

Loading of surgical meshes with SPIOs allows their precise visualization with MRI and guarantees an accurate in vivo assessment of their shrinkage. Our observation clearly indicates that shrinkage in vivo is remarkably less than shown by illustrated explantation measurements. Therefore, it is advisable to focus only on in vivo measurements for clinical conclusions. The findings show MR imaging of such meshes to be a reliable technique for checking the proper location of implanted meshes. This might help in assessing mesh-related complications more precisely and may allow for more precise planning and evaluation of revision surgery. This new technique could be applied as a routine checkup without radiation exposure, increasing the quality of both the mesh and the procedure, and thus could prevent unnecessary surgical revision.

Acknowledgments The authors thank Miss E. Krott for her assistance in handling the animals. This research project was financially supported by the German Federal Ministry of Education and Research (Ref. 01 EZ 0849).

Disclosures Drs. Nicolas Kühnert, Jens Otto, Nils A. Krämer, Hank C. W. Donker, Ioana Slabu, Martin Baumann, Christiane K. Kuhl, and Uwe Klinge have no conflicts of interest or financial ties to disclose.

Open Access This article is distributed under the terms of the Creative Commons Attribution Noncommercial License which permits any noncommercial use, distribution, and reproduction in any medium, provided the original author(s) and source are credited.

\section{References}

1. McGreevy JM, Goodney PP, Birkmeyer CM, Finlayson SRG, Laycock WS, Birkmeyer JD (2003) A prospective study comparing the complication rates between laparoscopic and open ventral hernia repairs. Surg Endosc 17:1778-1780

2. Andersen LPH, Klein M, Goegenur I, Rosenberg J (2009) Longterm recurrence and complication rates after incisional hernia repair with the open onlay technique. BMC Surg 9:6

3. Junge K, Klinge U, Rosch R, Klosterhalfen B, Schumpelick V (2002) Functional and morphologic properties of a modified mesh for inguinal hernia repair. World J Surg 26:1472-1480

4. Lomanto D, Iyer SG, Shabbir A, Cheah WK (2006) Laparoscopic versus open ventral hernia mesh repair: a prospective study. Surg Endosc 20:1030-1035

5. Schoenmaeckers EJP, van der Valk SBA, van den Hout HW, Raymakers JFTJ, Rakic S (2009) Computed tomographic measurements of mesh shrinkage after laparoscopic ventral incisional hernia repair with an expanded polytetrafluoroethylene mesh. Surg Endosc 23:1620-1623

6. Fischer T, Ladurner R, Gangkofer A, Mussack T, Reiser M, Lienemann A (2007) Functional cine MRI of the abdomen for the assessment of implanted synthetic mesh in patients after incisional hernia repair: initial results. Eur Radiol 17:3123-3129

7. Zogbi L, Portella AOV, Trindade MRM, Trindade EN (2010) Retraction and fibroplasia in a polypropylene prosthesis: experimental study in rats. Hernia J Hernias Abdom Wall Surg 14:291-298
8. Parra JA, Revuelta S, Gallego T, Bueno J, Berrio JI, Fariñas MC (2004) Prosthetic mesh used for inguinal and ventral hernia repair: normal appearance and complications in ultrasound and CT. Br J Radiol 77:261-265

9. Jenkins ED, Yom V, Melman L, Brunt LM, Eagon JC, Frisella MM, Matthews BD. Prospective evaluation of adhesion characteristics to intraperitoneal mesh and adhesiolysis-related complications during laparoscopic re-exploration after prior ventral hernia repair. Surg Endosc 24:3002-3007

10. Ferrari GC, Miranda A, Di Lernia S, Sansonna F, Magistro C, Maggioni D, Scandroglio I, Costanzi A, Franzetti M, Pugliese R (2008) Laparoscopic repair of incisional hernia: outcomes of 100 consecutive cases comprising 25 wall defects larger than $15 \mathrm{~cm}$. Surg Endosc 22:1173-1179

11. Conze J, Krones CJ, Schumpelick V, Klinge U (2007) Incisional hernia: challenge of reoperations after mesh repair. Langenbeck's Arch Surg Deutsche Gesellschaft Chir 392:453-457

12. Lienemann A, Sprenger D, Steitz HO, Korell M, Reiser M (2000) Detection and mapping of intraabdominal adhesions by using functional cine MR imaging: preliminary results. Radiology 217: 421-425

13. Zinther NB, Zeuten A, Marinovskij E, Haislund M, Wara P, FriisAndersen H (2010) Functional cine MRI and transabdominal ultrasonography for the assessment of adhesions to implanted synthetic mesh 5-7 years after laparoscopic ventral hernia repair. Hernia J Hernias Abdom Wall Surg 14:499-504

14. Mussack T, Fischer T, Ladurner R, Gangkofer A, Bensler S, Hallfeldt KK, Reiser M, Lienemann A (2005) Cine magnetic resonance imaging vs high-resolution ultrasonography for detection of adhesions after laparoscopic and open incisional hernia repair: a matched pair pilot analysis. Surg Endosc 19:1538-1543

15. Zinther NB, Zeuten A, Marinovskij E, Haislund Mt, FriisAndersen H (2010) Detection of abdominal wall adhesions using visceral slide. Surg Endosc 24:3161-3166

16. Kirchhoff S, Ladurner R, Kirchhoff C, Mussack T, Reiser MF, Lienemann A (2010) Detection of recurrent hernia and intraabdominal adhesions following incisional hernia repair: a functional cine MRI-study. Abdom Imaging 35:224-231

17. Kraemer NA, Donker HCW, Otto J, Hodenius M, Senegas J, Slabu I, Klinge U, Baumann M, Muellen A, Obolenski B, Guenther RW, Krombach GA (2010) A concept for magnetic resonance visualization of surgical textile implants. Invest Radiol 45:477-483

18. Kuhry E, Schwenk W, Gaupset R, Romild U, Bonjer J (2008) Long-term outcome of laparoscopic surgery for colorectal cancer: a Cochrane systematic review of randomised controlled trials. Cancer Treat Rev 34:498-504

19. Kingsnorth A, LeBlanc K (2003) Hernias: inguinal and incisional. Lancet 362:1561-1571

20. Klinge U, Junge K, Stumpf M, Ap APO, Klosterhalfen B (2002) Functional and morphological evaluation of a low-weight, monofilament polypropylene mesh for hernia repair. J Biomed Mater Res 63:129-136

21. García Ureña MA, Vega Ruiz V, Díaz Godoy A, Báez Perea JM, Marín Gómez LM, Carnero Hernández FJ, MAngel Velasco García (2007) Differences in polypropylene shrinkage depending on mesh position in an experimental study. Am J Surg 193: $538-542$

22. Harrell AG, Novitsky YW, Peindl RD, Cobb WS, Austin CE, Cristiano JA, Norton JH, Kercher KW, Heniford BT (2006) Prospective evaluation of adhesion formation and shrinkage of intraabdominal prosthetics in a rabbit model. Am Surg 72:808833 discussion $813-814$

23. Johnson EK, Hoyt CH, Dinsmore RC (2004) Abdominal wall hernia repair: a long-term comparison of Sepramesh and Dualmesh in a rabbit hernia model. Am Surg 70:657-661 
24. Jacob BP, Hogle NJ, Durak E, Kim T, Fowler DL (2007) Tissue ingrowth and bowel adhesion formation in an animal comparative study: polypropylene versus Proceed versus Parietex Composite. Surg Endosc 21:629-633

25. Burger JWA, Halm JA, Wijsmuller AR, ten Raa S, Jeekel J (2006) Evaluation of new prosthetic meshes for ventral hernia repair. Surg Endosc 20:1320-1325

26. Taupitz M, Schmitz S, Hamm B (2003) Superparamagnetic iron oxide particles: current state and future development. RoeFo Fortschr Geb Roentgenstr Nuklearmedizin 175:752-765

27. Kircher MF, Allport JR, Graves EE, Love V, Josephson L, Lichtman AH, Weissleder R (2003) In vivo high resolution threedimensional imaging of antigen-specific cytotoxic T-lymphocyte trafficking to tumors. Cancer Res 63:6838-6846

28. Junge K, Binneboesel M, Rosch R, Jansen M, Kaemmer D, Otto J, Schumpelick V, Klinge U (2009) Adhesion formation of a polyvinylidenfluoride/polypropylene mesh for intraabdominal placement in a rodent animal model. Surg Endosc 23:327-333
29. Berger D, Bientzle M (2009) Polyvinylidenfluoride: a suitable mesh material for laparoscopic incisional and parastomal hernia repair! A prospective, observational study with 344 patients. Hernia J Hernias Abdom Wall Surg 13:167-172

30. Conze J, Junge K, Weiss C, Anurov M, Oettinger A, Klinge U, Schumpelick V (2008) New polymer for intraabdominal meshes: PVDF copolymer. J Biomed Mater Res Part B Appl Biomater 87: 321-328

31. Dauendorffer JN, Bastuji-Garin S, Guéro S, Brousse N, Fraitag S (2009) Shrinkage of skin excision specimens: formalin fixation is not the culprit. Br J Dermatol 160:810-814

32. Blasdale C, Charlton FG, Weatherhead SC, Ormond P, Lawrence CM (2010) Effect of tissue shrinkage on histological tumour-free margin after excision of basal cell carcinoma. Br J Dermatol 162:607-610

33. Wheeler TL, Koohmaraie M (1994) Prerigor and postrigor changes in tenderness of ovine longissimus muscle. J Anim Sci $72: 1232-1238$ 